BULLETIN OF THE

AMERICAN MATHEMATICAL SOCIETY

Volume 77, Number 5, September 1971

\title{
INVARIANT POLYNOMIALS AND CONJUGACY CLASSES OF REAL CARTAN SUBALGEBRAS ${ }^{1}$
}

\author{
BY L. PREISS ROTHSCHILD
}

Communicated by M. Gerstenhaber, March 29, 1971

1. Introduction and statement of main result. For complex reductive Lie algebras the conjugacy of all the Cartan subalgebras by inner automorphisms plays a fundamental role in the structure and classification theory. The conjugacy theorem does not hold for real reductive Lie algebras, but each such algebra has only finitely many conjugacy classes of Cartan subalgebras. A complete classification of the conjugacy classes for all real simple Lie algebras was done by Kostant in 1955. (See Kostant [4], and Sugiura [7].) The proof of conjugacy of Cartan subalgebras in the complex case, requiring only the connectedness of the subset of regular semisimple elements [6, p. III-6, Theorem 2], suggests a relationship, in the real case, between the connected components of this subset and the conjugacy classes of Cartan subalgebras. Our purpose here is to establish such a relationship, using the invariant polynomials on the Lie algebra. We also show how this relates to the number of real zeros of polynomials.

Let $\mathrm{g}_{c}$ be a complex reductive Lie algebra, and $G_{c}$ any corresponding connected subgroup. We shall write $a \cdot x$ for the adjoint action of $a \in G_{c}$ on $x \in \mathfrak{g}_{c}$. Let $J$ be the ring of all polynomials on $\mathfrak{g}_{c}$ invariant under the adjoint action of $G_{c}$. By a famous result of Chevalley, there exist homogeneous polynomials $u_{1}, u_{2}, \cdots, u_{l}$, where $l$ is the rank of $\mathfrak{g}_{c}$, which are algebraically independent and generate $J$. (See Chevalley [1] and Helgason [2, pp. 429-434].)

$x \in \mathfrak{g}_{c}$ is called semisimple if ad $x$ is a diagonalizable endomorphism, where ad $x$ is the endomorphism of $\mathfrak{g}_{c}$ defined by ad $x \cdot y=[x, y]$ for any $y \in g_{c} . x$ is called regular if its orbit under the action of $G$ has maximal possible dimension. For any real form $\mathfrak{g}$ of $\mathfrak{g}_{c}$ let $G \subset G_{c}$ be the connected subgroup corresponding to g. $G$ acts as a group of automorphisms on $\mathfrak{g}$, and two Cartan subalgebras of $\mathfrak{g}$ are said to be conjugate if one is transformed into the other by some automorphism in $G$.

AMS 1970 subject classifications. Primary 17B20, 17B40, 22E15; Secondary 12D16, 53A55.

Key words and phrases. Lie algebras, semisimple Lie groups, Cartan subalgebras, invariant polynomials.

1 This work was supported by an N.S.F. Graduate Fellowship.

Copyright (C) American Mathematical Society 1971 
THEOREM. Let $u: g_{c} \rightarrow C^{l}$ be defined by $u(x)=\left(u_{1}(x), u_{2}(x), \cdots\right.$, $\left.u_{l}(x)\right)$, and let $\mathfrak{g}_{c}^{*} \subset g_{c}$ be the subset of regular semisimple elements. For any real form $\mathfrak{g}$ of $\mathfrak{g}_{c}$ the restriction of $u$ to $\mathrm{g}$ gives a 1-1 correspondence between the conjugacy classes of Cartan subalgebras of $g$ and the connected components of the image $u\left(\mathfrak{g}_{c}^{*} \cap \mathfrak{g}\right)$.

EXAmpLE. Let $\mathfrak{g}=\mathfrak{g} \mathfrak{l}(2, R)$, the set of all $2 \times 2$ real matrices of trace $0 . \mathfrak{g}$ has two conjugacy classes of Cartan subalgebras, represented by the subalgebras

$$
\mathfrak{h}_{1}=\left\{\left(\begin{array}{rr}
a & 0 \\
0 & -a
\end{array}\right): a \in R\right\} \quad \text { and } \quad \mathfrak{h}_{2}=\left\{\left(\begin{array}{rr}
0 & b \\
-b & 0
\end{array}\right): b \in R\right\}
$$

The determinant function, det, generates the algebra of invariant polynomials. Since any nonsingular $x \in \mathfrak{g}$ is regular, $\operatorname{det}\left(\mathfrak{g} \cap \mathfrak{g}_{c}^{*}\right)=R$ $-\{0\}$, which is the union of the connected components $R^{-}$ $=\operatorname{det}\left(\mathfrak{h}_{1}-\{0\}\right)$ and $R^{+}=\operatorname{det}\left(\mathfrak{h}_{2}-\{0\}\right)$.

2. Outline of the proof of the theorem. The main results needed for the proof of the theorem are the following four lemmas. For a Cartan subalgebra $\mathfrak{h} \subset \mathfrak{g}$, we write $\mathfrak{h}^{*}$ for $\mathfrak{h} \cap \mathfrak{g}_{\mathfrak{c}}^{*}$.

Lemma 1. For any $x, y \in \mathfrak{g}_{c}, u(x)=u(y)$ iff $x=a \cdot y$ for some $a \in G_{c}$.

For proof, see Helgason [2, p. 433] or Kostant [3, Proposition 10].

Lemma 2. If $x, y \in \mathfrak{g}$ are semisimple and satisfy $x=a \cdot y$ for some $a \in G_{c}$, then there exists $a_{0} \in G$ such that $a_{0} \cdot x, y$ are both in some Cartan subalgebra $\mathfrak{h} \subset \mathfrak{g}$.

Lemma 2 is proved in Rothschild [5].

Lemmas 1 and 2 together imply that $u\left(\mathfrak{h}_{1}^{*}\right) \cap u\left(\mathfrak{h}_{2}^{*}\right)=\varnothing$ for any Cartan subalgebras $\mathfrak{h}_{1}, \mathfrak{h}_{2}$ of $\mathfrak{g}$ which are not conjugate. The following result is needed to show that $u\left(\mathfrak{h}_{1}^{*}\right) \cup u\left(\mathfrak{h}_{2}^{*}\right)$ consists of at least two components.

Lemma 3. The differentials $d u_{1}, d u_{2}, \cdots, d u_{l}$ are linearly independent at any regular semisimple $x \in \mathrm{g}_{c}$.

A proof may be found in Kostant [3, Theorem 9] or Varadarajan [8].

Finally, a completely new result must be used to show that $u\left(\mathfrak{h}_{1}^{*}\right) \cup u\left(\mathfrak{h}_{2}^{*}\right)$ consists of at most two components.

Lemma 4. There exists a connected subset $C \subset \mathfrak{h}^{*}$ such that for any $x \in \mathfrak{g}^{*}$ there exists $y \in C$ and $a \in G_{c}$ with $a \cdot x=y$.

Lemmas 1 and 4 show that $u\left(\mathfrak{b}^{*}\right)$ is connected. 
REMARK. In general the number of connected components of $\mathfrak{g}^{*}$ greatly exceeds the number of conjugacy classes of Cartan subalgebras of $\mathfrak{g}$. A formula for the exact number of such connected components will be given with the details of the proof of the theorem above.

3. Relationship to real zeros of polynomials. The Lie algebra $\mathfrak{g}=\mathfrak{g l}(n, R)$, all $n \times n$ matrices, has [ $n / 2]$ distinct conjugacy classes of Cartan subalgebras, where $[n / 2]$ is the greatest integer in $n / 2$. These classes correspond to the possible numbers of real roots of the characteristic polynomial of a real matrix. The coefficients of the characteristic polynomial may be taken for $u_{1}, u_{2}, \cdots, u_{n}$, and the regular semisimple elements in $g$ are those diagonalizable matrices whose characteristic polynomial polynomials have no repeated roots. Furthermore, $u(\mathrm{~g})=\boldsymbol{R}^{n}$; that is, any monic polynomial of degree $n$ is the characteristic polynomial for some $x \in \mathfrak{g}$. Finally, any point $\left(a_{0}, a_{1}, \cdots, a_{n-1}\right) \in R^{n}$ may be identified with the monic polynomial $X^{n}+a_{n-1} X^{n-1}+\cdots+a_{0}$. This allows application of the theorem to prove the following result on the roots of real polynomials, which can also be proved directly.

CoRollary. Let $V \subset R^{n}$ be the points corresponding to polynomials with repeated roots, and let $C$ be any connected component of $R^{n}-V$. Then any two points in $C$ correspond to polynomials having the same number of real roots. Conversely, two points in different components correspond to polynomials having different numbers of real roots.

\section{REFERENCES}

1. C. Chevalley, Invariants of finite groups generated by reflections, Amer. J. Math. 77 (1955), 778-782. MR 17, 345; 1436.

2. S. Helgason, Differential geometry and symmetric spaces, Pure and Appl. Math., vol. 12, Academic Press, New York, 1962. MR 26 \#2986.

3. B. Kostant, Lie group representations on polynomial rings, Amer. J. Math. 85 (1963), 327-404. MR $28 \# 1252$.

4. - On the conjugacy of real Cartan subalgebras. I, Proc. Nat. Acad. Sci. U.S.A. 41 (1955), 967-970. MR 17, 509.

5. L. Rothschild, Orbits in a real reductive Lie algebra, Trans. Amer. Math. Soc. (to appear).

6. J.-P. Serre, Algèbres de Lie semi-simples complexes, Benjamin, New York, 1966. MR 35 \#6721.

7. M. Sugiura, Conjugate classes of Cartan subalgebras in real semisimple Lie algebras, J. Math. Soc. Japan 11 (1959), 374-434. MR 26 \#3827.

8. V. S. Varadarajan, On the ring of invariant polynomials on a semisimple Lie algebra, Amer. J. Math. 90 (1968), 308-317. MR 37 \#1529.

Massachusetts Institute of Technology, Cambridge, Massachusetts 02139

Tufts University, Medford, Massachusetts 02155 